11,12

\title{
Рентгенографические исследования реальной структуры ортобората иттрия $\mathrm{YBO}_{3}$
}

\author{
() И.М. Шмытько, Г.Р. Ганеева \\ Институт физики твердого тела РАН, \\ Черноголовка, Россия \\ E-mail: shim@issp.ac.ru
}

(Поступила в Редакцию 18 мая 2016 г.)

\begin{abstract}
Проведены детальные рентгеновские исследования изменения структуры ортобората иттрия в процессе последовательных высокотемпературных изотермических отжигов исходно аморфного прекурсорного состояния. Установлено, что измеренное при комнатной температуре распределение интенсивности дифракционных рефлексов $\mathrm{YBO}_{3}$, полученных на начальных этапах кристаллизации, соответствует известной низкотемпературной фазе фатерита с симметрией пространственной группы (пр. гр.) симметрии $P 6_{3} / m$, а после последовательности высокотемпературных отжигов оно трансформируется в распределение, известное для модификации фатерита с симметрией пр. гр. $P 6_{3} / m m c$ с теми же параметрами решетки. Полученный результат объяснен на основе учета сферичности рентгеновских волн и связан с изменением формы кристаллитов от шарообразной при низкотемпературных отжигах к гантелеобразной при высокотемпературных отжигах.

В результате экспериментов, проведенных in situ при $1250^{\circ} \mathrm{C}$, установлено, что исходная низкотемпературная гексагональная ячейка фатерита выше $1000^{\circ} \mathrm{C}$ трансформируется в моноклинную ячейку.
\end{abstract}

Работа выполнена в рамках научного плана ИФТТ РАН.

\section{1. Введение}

В настоящее время большое внимание уделяется изучению структурного состояния и фазовых трансформаций наноскопических систем при изменении размера кристаллитов. Интересными в этом отношении являются простые и сложные оксиды и фториды редкоземельных элементов. Интерес к оксидам и фторидам редкоземельных элементов обусловлен прежде всего тем, что многие из них обладают прекрасными сцинтилляционными и люминофорными характеристиками. Нами ранее были проведены детальные структурные исследования ряда сложных редкоземельных оксидных и фторидных соединений, синтезированных из аморфного прекурсорного состояния [1-14]. При этом было показано, что их структура сильно зависит от размера кристаллитов. В частности, рентгеновские исследования показали, что на первых этапах кристаллизации этих соединений из аморфных прекурсоров отдельные нанокристаллиты состоят из фазы ядра и изоморфной ей поверхностной фазы с бол́ышим периодом решетки core-shell структура $[4,5,7,13]$. Кроме того, были открыты циклические перестройки структуры с ростом размера кристаллитов, неизвестные для микрокристаллического состояния и обусловленные для наноразмеров повышенной энергией поверхностных атомов по сравнению с энергией объемных $[1,11,14]$. Был также открыт эффект структурного заражения, когда малые добавки легирующих атомов приводили к образованию структур, свойственных оксидам легирующего элемента, но не образующихся в матричном составе без легирования $[1,8]$. Впервые для твердофазного синтеза сложных соединений был зарегистрирован эффект инициирования фазообразования в процессе непрерывного повышения температуры синтеза, приводящий в отдельных случаях к сокращению времени синтеза в несколько раз по сравнению с имеющим место при ступенчатом изотемпературном отжиге $[2,6,7]$. Было также зафиксировано образование в нанокристаллическом состоянии новых структур, неизвестных в микрокристаллическом состоянии [12].

В отмеченных выше исследованиях не возникал вопрос о достоверности определения симметрии фаз исследованных соединений, известных из литературных данных. Такой вопрос, однако, существует для ортобората иттрия $\mathrm{YBO}_{3}$. До сих пор возникают разногласия по поводу его структуры при комнатной температуре как таковой и структуры его высокотемпературной фазы [15-25]. Так, на основе базы структурных данных PDF-2 в обоих случаях борат иттрия имеет гексагональную решетку фатерита и пространственную группу (пр. гр.) симметрии $P 6_{3} / m$ (№ 00-088-0356 в базе данных PDF-2) для низкотемпературной фазы и пр. гр. $P 6_{3} / m m c$ (№ 00-074-1929 в базе данных PDF-2) для высокотемпературной фазы. Это же утверждается и в работах [15-23]. В то же время в работах [24,25] сделано заключение, что реальная структура $\mathrm{YBO}_{3}$ имеет моноклинную кристаллическую ячейку и пр. гр. симметрии $C 2 / c$. Более того, в работе [24] приводятся литературные данные о структурных состояниях с пр. гр. симметрии $P \overline{6} c 2, R 32, C 2 / c$. Одной из причин такого несоответствия может быть зависимость структурного состояния от размера кристаллитов, обусловливающая, в частности, циклические перестройки структуры с ростом размера кристаллитов $[1,11,14]$. Кроме того, на структурные данные предположительно может оказы- 
вать влияние не только размер, но и форма кристаллитов. Наш метод синтеза боратов редких земель из аморфных прекурсоров при повышенных температурах отжига позволяет менять как размер кристаллитов от нанодо микроразмера, так и их форму, что дает основание надеяться на получение ответа на поставленный вопрос о реальном структурном состоянии ортобората иттрия, положенный в основу проводимых нами исследований.

\section{2. Экспериментальные методы и образцы}

Аморфные прекурсоры бората иттрия были синтезированы при обменном взаимодействии нитратов иттрия с борной кислотой в водной среде. Для синтеза использовались реактивы квалификации хч. Водный раствор нитрата иттрия получался либо его растворением в минимальном количестве воды, либо растворением соответствующего количества оксида в $40 \%$ азотной кислоте при кипячении. Далее водный раствор нитрата смешивался с водным раствором эквимольного количества борной кислоты. При перемешивании добавлялся водный раствор аммиака до достижения щелочной реакции среды $(\mathrm{pH}=9.5-10)$. Затем полученный порошок отделяли либо на плотном стеклянном фильтре, либо центрифугированием, промывали несколько раз водой, сушили на воздухе при $50-700 \mathrm{C}$ в течение $20 \mathrm{~h}$ и уплотнившуюся компактную массу растирали в агатовой ступке.

Твердофазный синтез $\mathrm{YBO}_{3}$ осуществлялся путем последовательных изотермических отжигов аморфного прекурсора в течение $1-4 \mathrm{~h}$ в лабораторной печи SNOL-6.7/1300 на воздухе при температурах 600, 700, $800,900^{\circ} \mathrm{C}$ и т.д. После очередного отжига образец извлекался из печи для быстрого охлаждения на воздухе и определения его структурного состояния. Если после очередного отжига наблюдалось заметное изменение дифракционных спектров, температурный шаг последующего отжига уменьшался до нескольких десятков градусов или проводился повторный отжиг при той же температуре. Кроме того, рентгеновские исследования проводились in situ в высокотемпературной рентгеновской камере HTK-2000 (ANTON PAAR), позволяющей поддерживать заданную температуру в интервале $100-1300^{\circ} \mathrm{C}\left( \pm 1.0^{\circ} \mathrm{C}\right)$.

Структурное состояние образцов регистрировалось на рентгеновском дифрактометре D500 (Siemens) с использованием схемы Брэгга-Брентано. Использовалось монохроматизированное выходным графитовым монохроматором $\mathrm{Cu} K_{\alpha}$-излучение. Изменение формы кристаллитов регистрировалось на сканирующем электронном микроскопе (SEM) SUPRA II.

\section{3. Результаты и обсуждение}

На рис. 1 показаны дифракционные спектры бората иттрия $\mathrm{YBO}_{3}$ для исходного прекурсорного состояния

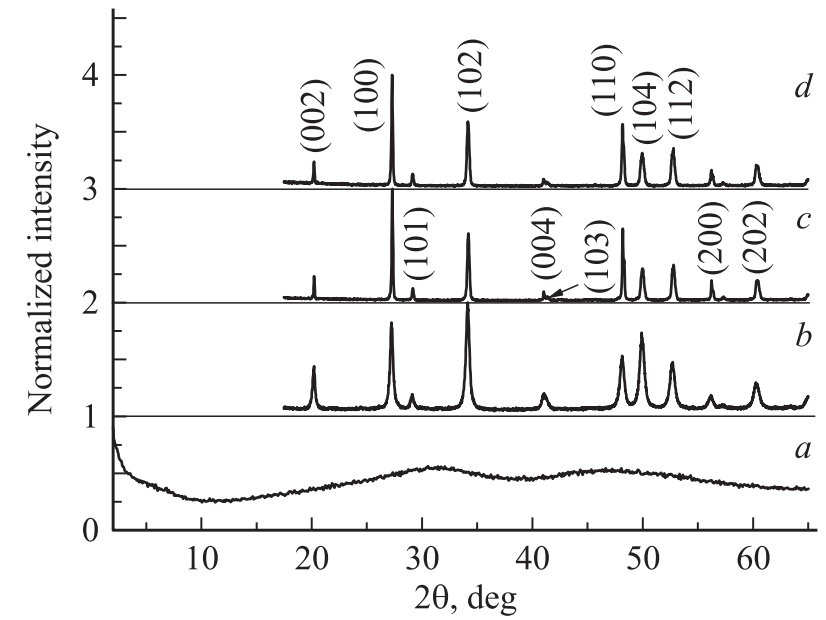

Рис. 1. Дифракционные спектры $\mathrm{YBO}_{3} . a-$ исходное аморфное прекурсорное состояние, $b-$ образец последовательно отожжен при $T=600$ и $700^{\circ} \mathrm{C}$ по $2 \mathrm{~h}, c-$ образец последовательно отожжен через каждые $100^{\circ} \mathrm{C}$ от 600 до $1200^{\circ} \mathrm{C}$ по $2 \mathrm{~h}$ в каждой температурной точке, $d-$ образец, отожженнный при $1200^{\circ} \mathrm{C}$, дополнительно отожжен при $700^{\circ} \mathrm{C}$ в течение $12 \mathrm{~h}$ и при $535^{\circ} \mathrm{C}$ в течение $25 \mathrm{~h}$.

(спектр $a$ ), после последовательности отжигов при $T=600$ и $700^{\circ} \mathrm{C}$ по $2 \mathrm{~h}($ спектр $b)$ и после череды последующих отжигов через каждые $100^{\circ} \mathrm{C}$ до конечной температуры, равной $1200^{\circ} \mathrm{C}$ (спектр $c$ ). Все спектры были получены при комнатной температуре после соответствующего отжига образца в лабораторной печи SNOL$6.7 / 1300$ на воздухе. Из сравнения спектров $b$ и $c$ хорошо видно, что относительные интенсивности многих рефлексов значительно изменились. Если исходить из штрихдиаграмм базы данных PDF-2 (рис. 2), то спектр $b$ на рис. 1 соответствует низкотемпературной фазе фатерита (пр. гр. $\left.P 6_{3} / m(176)\right)$, а спектр $c$ - высокотемпературной фазе фатерита (пр. гр. $\left.P 6_{3} / m m c(194)\right)$, в которую, согласно литературным данным, низкотемпературный фатерит переходит вблизи $1000^{\circ} \mathrm{C}$ [15-23]. Однако, согласно калориметрическим измерениям, проведенным в работе [21], высокотемпературная фаза не может сохраняться при комнатной температуре. Она испытывает обратный фазовый переход в низкотемпературную фатеритную модификацию, хотя и со значительным температурным гистерезисом, но не ниже $600^{\circ} \mathrm{C}$ даже при быстром охлаждении образца. Тем не менее, предполагая, что кинетика обратного фазового перехода из высокотемпературной фазы фатерита в низкотемпературную может в наших экспериментах быть по какой-то причине замедленной, для образца, отожженного при $1200^{\circ} \mathrm{C}$, мы провели дополнительные отжиги при $700^{\circ} \mathrm{C}$ в течение $12 \mathrm{~h}$ и затем еще при $535^{\circ} \mathrm{C}$ в течение $25 \mathrm{~h}$. Никаких заметных изменений в распределении интенсивностей рефлексов после каждого среднетемпературного отжига не наблюдалось (спектр $d$ на рис. 1). Это обстоятельство ставит вопрос о фазовых состояниях, отображенных на рис. 1. 

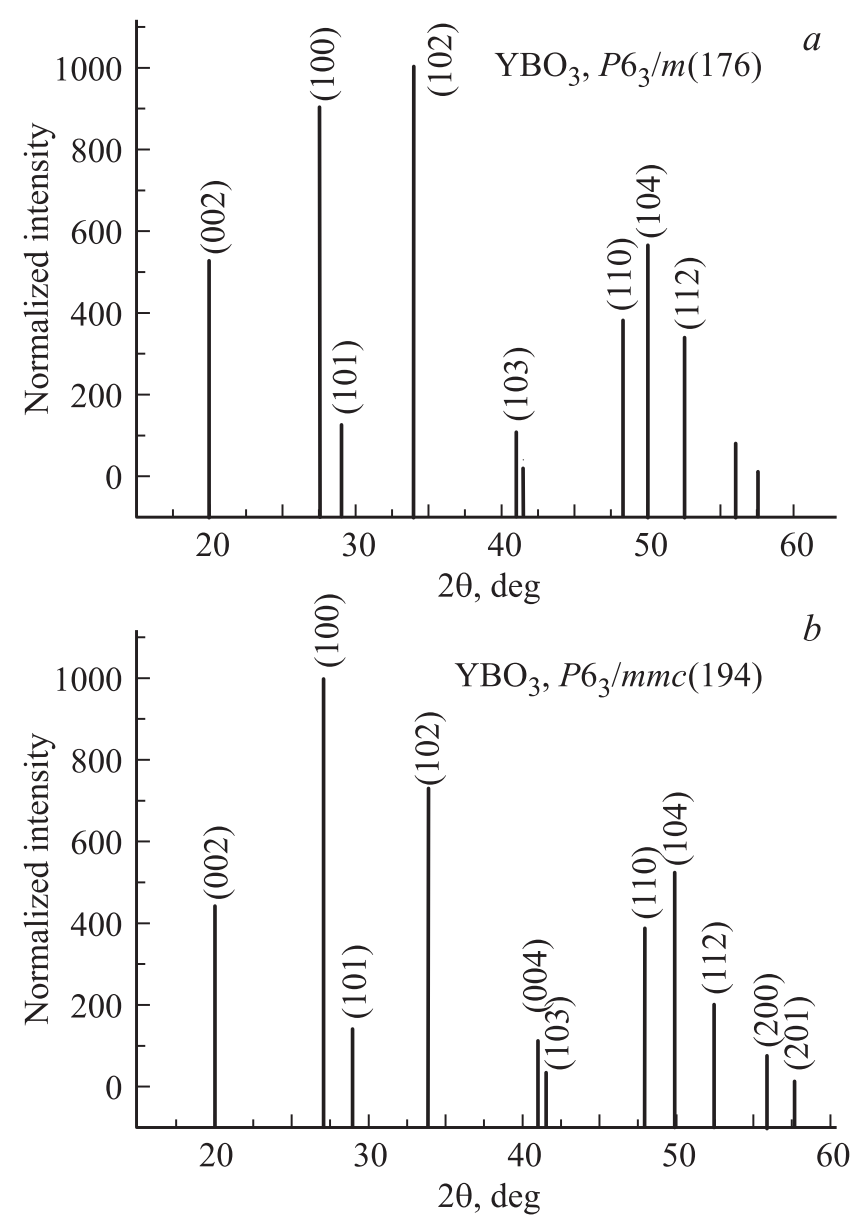

Рис. 2. Штрихдиаграммы для низкотемпературной (a) и высокотемпературной $(b)$ фаз фатерита $\mathrm{YBO}_{3}$ (согласно базе структурных данных PDF-2)

Можно было бы предположить, что первой кристаллической фазой, синтезированной из аморфного прекурсорного состояния, является не низкотемпературный, а высокотемпературный фатерит. Такое состояние возможно. Например, для $\mathrm{LuBO}_{3}, \mathrm{LaBO}_{3}$ и $\mathrm{Eu}_{2}\left(\mathrm{MoO}_{4}\right)_{3}$ первыми фазами, синтезированными на начальных этапах кристаллизации из аморфных прекурсоров, являются высокотемпературные фазы $[1,11,14]$. Если это выполняется и для $\mathrm{YBO}_{3}$, высокотемпературная фаза фатерита, образованная на первых этапах кристаллизации из аморфного состояния и отображенная на спектре $b$ (рис. 1), после разрастания кристаллитов при повышенных температурах отжига должна переходить в низкотемпературную фазу фатерита, равновесную при комнатной температуре, отображенную на спектре $c$ (рис. 1). В этом случае неизменность дифракционных спектров $\mathrm{YBO}_{3}$ при комнатной температуре даже после дополнительных среднетемпературных отжигов была бы связана с нахождением образца уже в равновесной низкотемпературной фазе. Для проверки такого предположения были проведены высокотемпературные исследования $\mathrm{YBO}_{3}$ in situ.
На рис. 3 показаны нормированные на пиковую интенсивность рефлекса (100) спектры $\mathrm{YBO}_{3}$, полученные для одного и того же образца при разных условиях отжига. Спектр $a$ измерен при комнатной температуре после отжига порошкообразного образца при температуре $1200^{\circ} \mathrm{C}$. Далее полученный образец был спрессован в таблетку при нагрузке, равной $6.4 \cdot 10^{3} \mathrm{~kg} / \mathrm{cm}^{2}$, помещен в высокотемпературную рентгеновскую камеру HTK-2000 (ANTON PAAR) и исследован в вакууме in situ при температуре $1250^{\circ} \mathrm{C}$ (спектр b). Спектр $c$ зарегистрирован в той же камере в вакууме после понижения температуры образца до комнатной. Сравнение спектров показывает, что соотношение интенсивностей всех базисных рефлексов практически не изменилось. Последнее обстоятельство и могло бы быть интерпретировано как отсутствие обратного фазового перехода из высокотемпературной фазы фатерита в низкотемпературную. Однако это не так.

На рис. 4 более детально показаны участки дифракционных спектров для образца, исследованного в высокотемпературной вакуумной камере in situ при $1250^{\circ} \mathrm{C}$, и образца, исследованного при комнатной температуре, но предварительно отожженного на воздухе при $1200^{\circ} \mathrm{C}$ в печи SNOL-6.7/1300. Штриховыми вертикальными линиями показаны положения штрихдиаграммы фазы фатерита для $\mathrm{YBO}_{3}$ из базы данных PDF-2. Смещение рефлексов относительно базисной штрихдиаграммы обусловлено как изменением температуры, так и изменением положения образца по высоте относительно падающего пучка при высокой температуре нагревателя камеры, который одновременно служил и держателем образца. К сожалению, производить in situ при температуре юстировку образца в камере НTK-2000 не представлялось возможным из-за ее конструктивных особенностей. Однако это обстоятельство не препятствует и даже

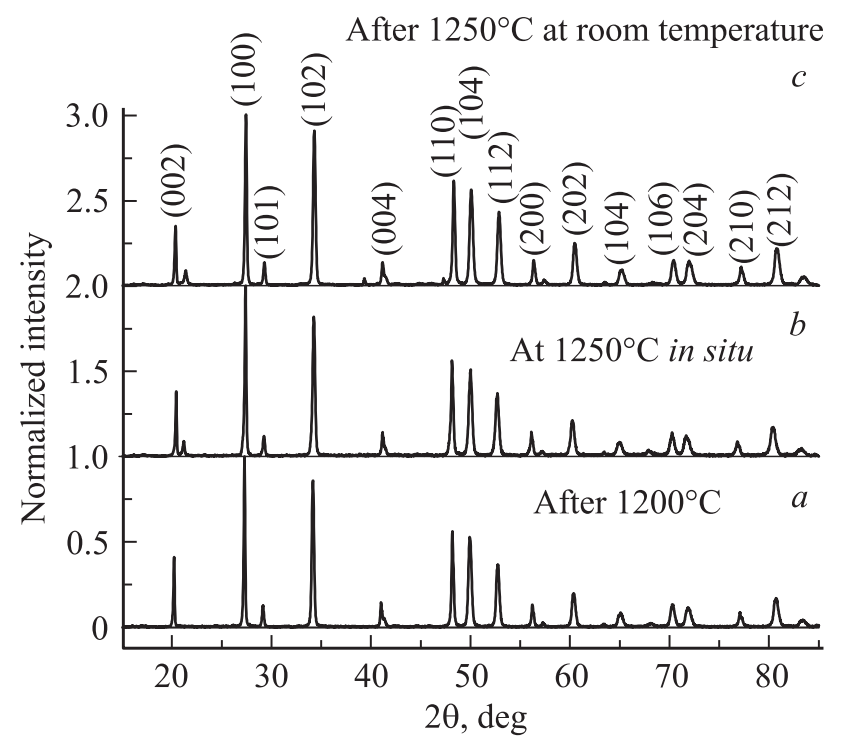

Рис. 3. Рентгеновские дифракционные спектры $\mathrm{YBO}_{3}$ одного и того же образца, измеренные при различных условиях отжига (см. текст). 

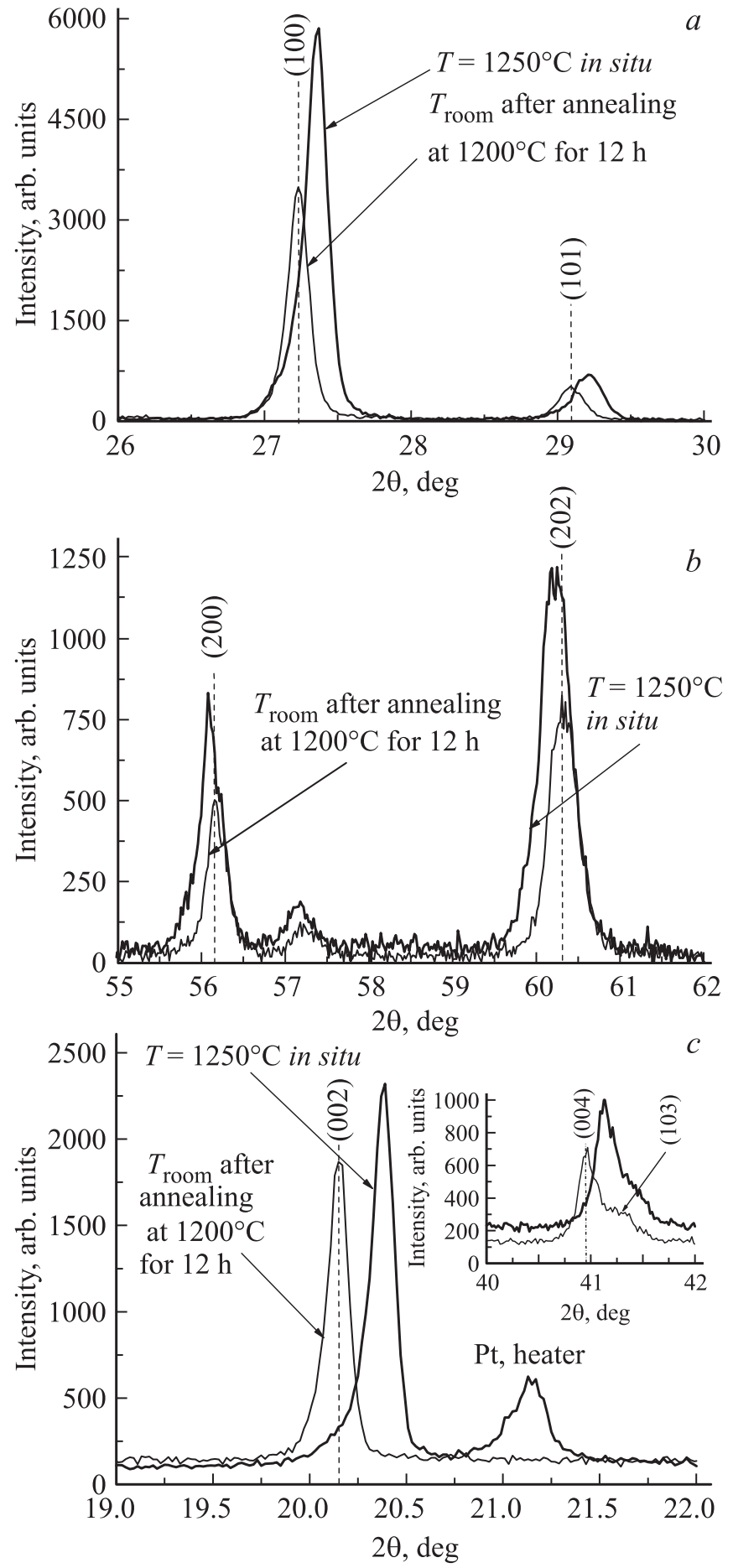

Рис. 4. Детальное отображение базисных рефлексов рентгеновских дифракционных спектров $\mathrm{YBO}_{3}$, показанных на рис. 3 (см. текст).

помогает проследить за относительным изменением положений базисных рефлексов $(h 00)$ и $(00 l)$ при комнатной температуре и при съемке in situ при $T=1250^{\circ} \mathrm{C}$. Из сравнения положений рефлексов (100) и (200) на рис. 4, $a$ и $b$ хорошо видно, что при $T=1250^{\circ} \mathrm{C}$ они сдвинуты относительно базисных положений в разные стороны. В то же время рефлексы (002) и (004) на рис. 4, с сдвинуты в одну сторону. Сдвиг „высокотемпературных“ рефлексов в разные стороны относительно порядков отражения базисных рефлексов (100) и (200) означает, что эти рефлексы уже не являются порядками отражения и, следовательно, отображают фазовый переход выше $1000^{\circ} \mathrm{C}$ в новую низкосимметричную фазу. В то же время из положения „высокотемпературных“ рефлексов, параллельных базисным отражениям (002) и (004), следует, что они продолжают оставаться порядками отражений. Последнее означает, что базисное направление $[00 l]$ сохранило свою ориентацию и в высокотемпературной фазе, а основные изменения произошли в базисной плоскости $a b$ исходной гексагональной модификации. Отсюда мы делаем заключение, что наиболее вероятной высокотемпературной фазой $\mathrm{YBO}_{3}$ является фаза с моноклинной ячейкой.

Таким образом, приведенные результаты наглядно показывают, что в кристаллах ортобората иттрия действительно реализуется моноклинная модификация, но она образуется при высокой температуре. При этом малое изменение параметров решетки в базисной плоскости $a b$ исходной ячейки указывает на малые смещения атомов в ней при высокотемпературном фазовом переходе. Это в свою очередь приводит к малому изменению в распределении интенсивностей рефлексов высокотемпературной фазы по сравнению с интенсивностями рефлексов базисной модификации, что и отображено на рис. 3.

Остается вопрос, чем же тогда обусловлено такое сильное перераспределение интенсивности рефлексов при комнатной температуре после высокотемпературных отжигов образца, отображенное на рис. 1. Единственными параметрами, изменяющимися при последовательных отжигах образца, являются размер и форма кристаллитов. Для установления влияния размера кристаллитов на распределение соотношения интенсивностей рентгеновских рефлексов были проведены детальные измерения пиковой и интегральной интенсивностей рефлексов после последовательных отжигов одного и того же образца. Такие изменения показаны на рис. 5 для отражения (102). Из рис. 5, $a$ видно, что интегральная интенсивность сначала растет с температурой отжига до $900^{\circ} \mathrm{C}$, затем несколько снижается в области возможного фазового перехода при $1000^{\circ} \mathrm{C}$, а потом снова возрастает с повышением температуры отжига до $1100^{\circ} \mathrm{C}$. Далее при температуре отжига $1200^{\circ} \mathrm{C}$ происходит падение интегральной интенсивности, которое является тем более сильным, чем больше время отжига образца. Аналогичное изменение наблюдается и для пиковой интенсивности (рис. 5, $b$ ). Различие лишь в том, что температурная точка фазового перехода для пиковой интенсивности выделена менее сильно, чем для интегральной интенсивности. Полученный результат однозначно указывает на влияние размера кристаллитов на распределение интенсивностей рефлексов. Действительно, если бы „перекачка“ интенсивности базисных рефлексов выше температуры $1000^{\circ} \mathrm{C}$ была бы обусловлена только фазовым переходом в высокотемпературную фазу, то в 

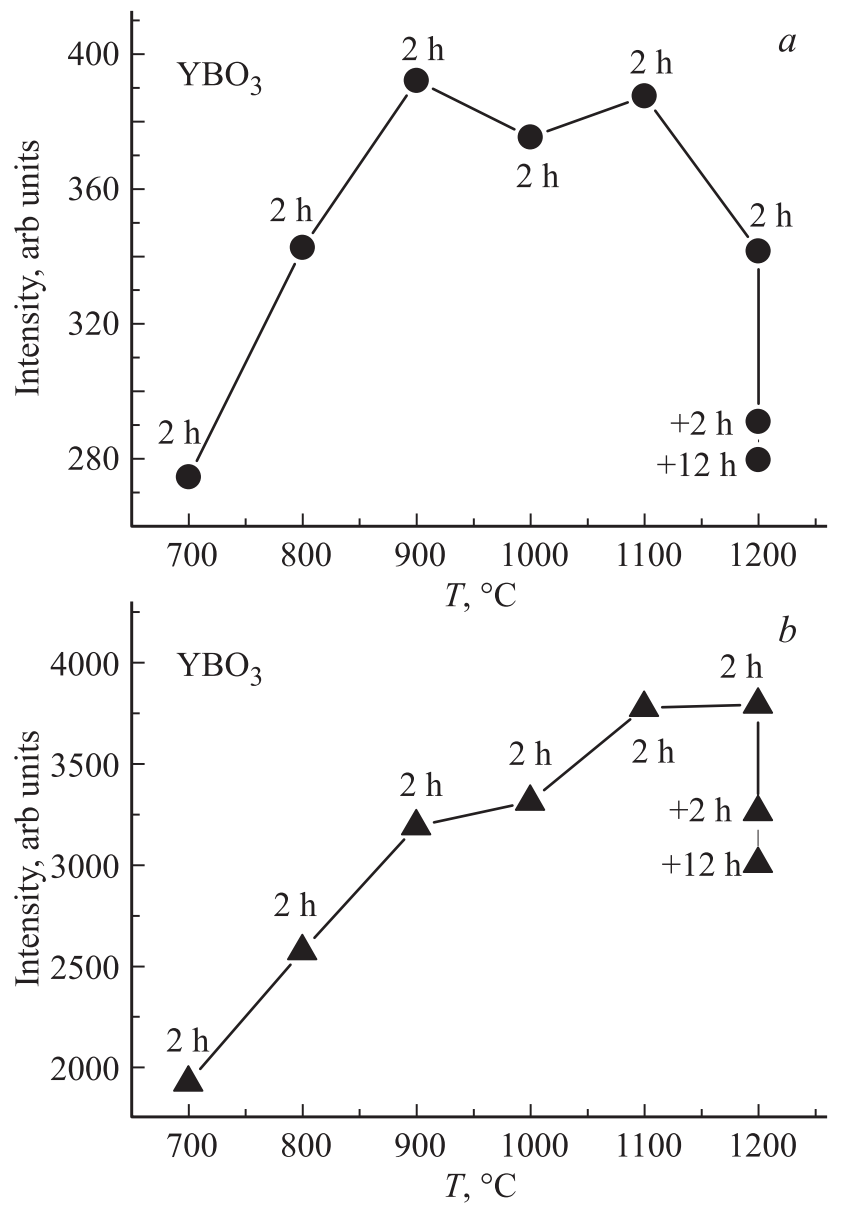

Рис. 5. Зависимость интегральной интенсивности рефлекca $(102)$ от температуры и времени отжига $(a)$ и зависимость пиковой интенсивности того же рефлекса $(b)$.

этом случае относительное распределение интенсивности рентгеновских рефлексов не должно было бы зависеть от последовательности отжигов, а определялось бы только симметрией высокотемпературной фазы. Вывод о влиянии размеров кристаллитов на интенсивности рефлексов подтверждается и изменением соотношения интенсивностей базисных рефлексов для двух разных образцов, испытавших различные последовательности отжигов (рис. 1 и 3 ).

Ясно, что если кристаллиты имеют сферическую форму, то независимо от их диаметра относительного перераспределения интенсивностей базисных рефлексов не произойдет в силу симметрии формы. Такое перераспределение возможно, если для разных кристаллографических направлений размер кристаллитов будет сильно различаться. Последнее и отображает влияние размера и формы кристаллитов, зависящих от режимов отжига, на соотношение интенсивностей базисных рефлексов. Действительно, на SEM-изображениях, полученных методом электронной сканирующей микроскопии (рис. 6), хорошо видно, что при низких температурах отжига наночастицы $\mathrm{YBO}_{3}$ имеют форму, близкую к сферической, а при температуре отжига, равной $1200^{\circ} \mathrm{C}$, кристаллиты приобретают форму гантелей с размером в несколько микрометров вдоль длинной оси.

Изменение формы кристаллитов в зависимости от температуры отжига также хорошо отображается на значениях полуширин различных рефлексов. На рис. 7 показано изменение полуширин рефлексов (а следова-

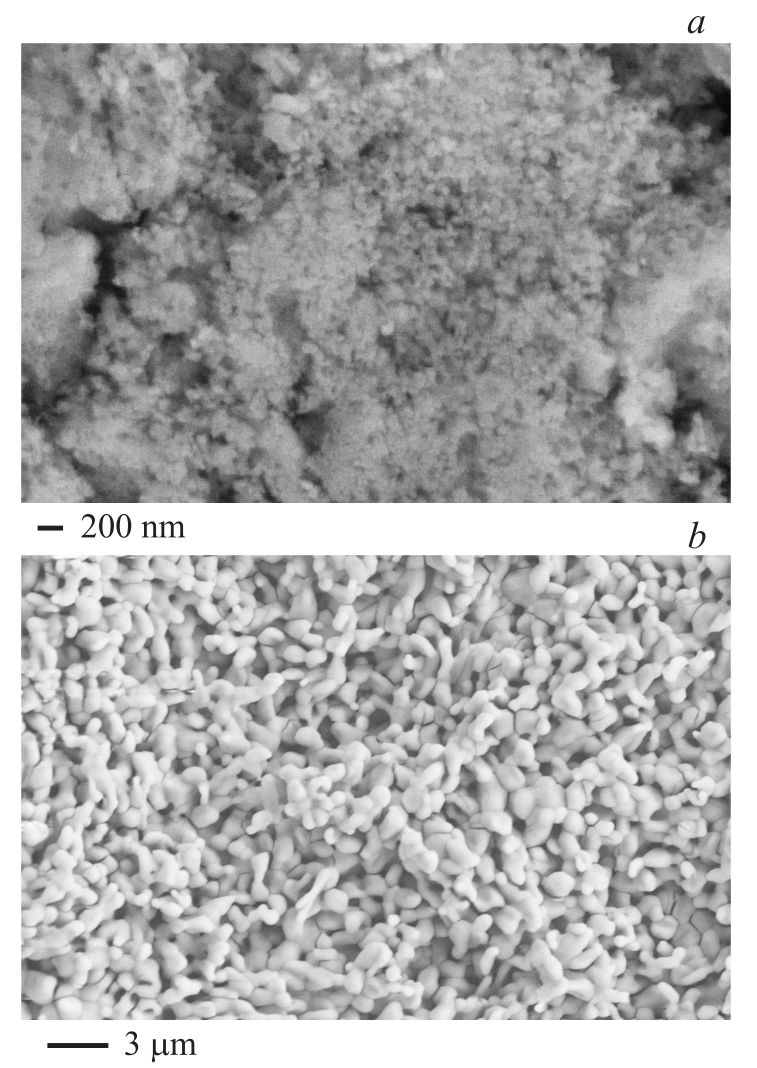

Рис. 6. SEM-изображения $\mathrm{YBO}_{3}$ после отжига прекурсора при $800^{\circ} \mathrm{C}$ в течение $2 \mathrm{~h}(a)$ и последующего отжига при $1200^{\circ} \mathrm{C}$ в течение $16 \mathrm{~h}(b)$.

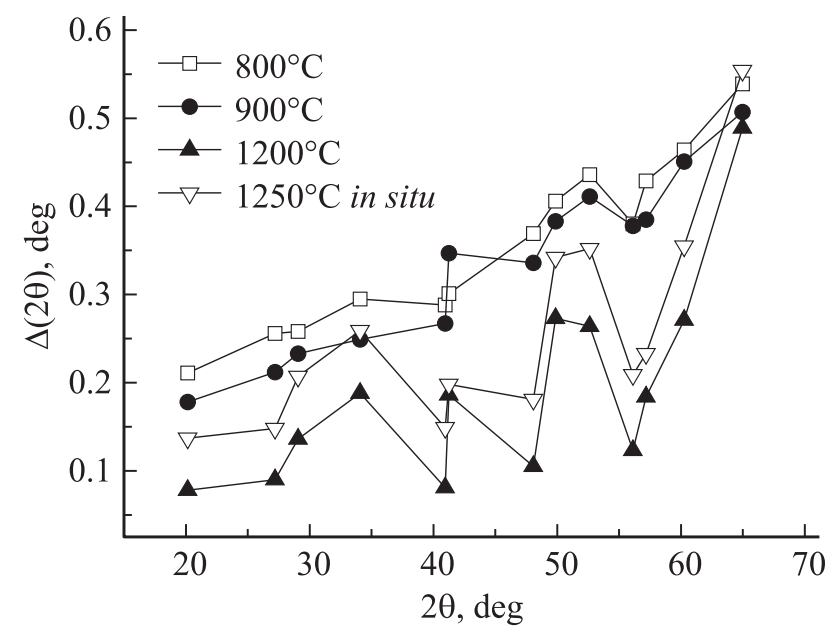

Рис. 7. Угловая зависимость полуширины рефлексов $(h k l)$ $\mathrm{YBO}_{3}$ при различных температурах отжига образца, выраженной в единицах углов дифракции. 


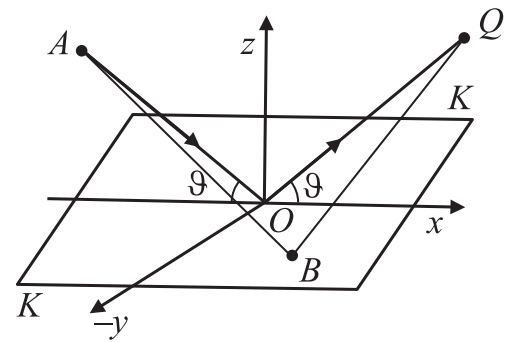

Рис. 8. Схема хода рентгеновских лучей для вычисления геометрии зон Френеля.

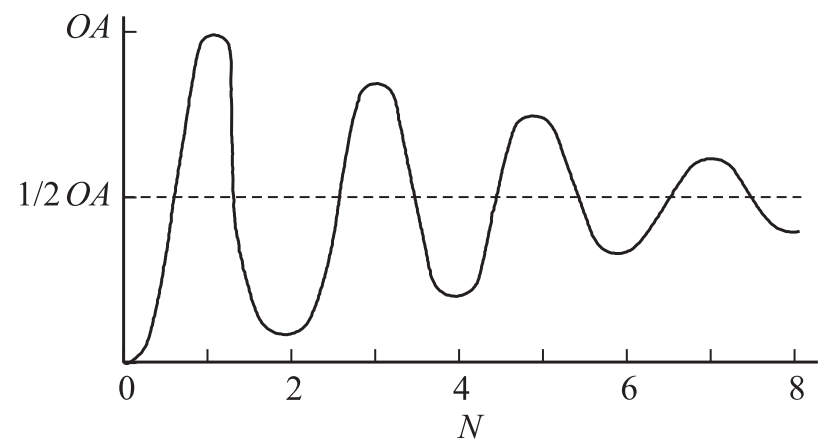

Рис. 9. Зависимость величины результирующей амплитуды рассеяния $O A$ от числа открытых зон Френеля $N$.

тельно, и размеров кристаллитов), выраженных в единицах углов дифракции $2 \theta$, для разных кристаллографических направлений в зависимости от температуры отжига. Хорошо видно, что если при температурах отжига, меньших $1000^{\circ} \mathrm{C}$, зависимости полуширин от угла дифракции отражения представляют собой относительно монотонные функции, то выше $1200^{\circ} \mathrm{C}$ значения полуширин резко изменяются для разных кристаллографических направлений. Последнее указывает на кардинальные изменения формы кристаллитов с увеличением температуры и времени отжига.

Полученный результат может быть объяснен на основе классической теории изменения интенсивности рассеяния света в зависимости от количества перекрытых объектом зон Френеля для сферической падающей волны. Согласно теории рассеяния, изложенной в книге Ивероновой и Ревкевич [26], разность хода $\Delta R$ между центральным лучом, прошедшим путь фокус рентгеновской трубки-кристалл-точка наблюдения, и лучом, рассеянным точкой $B$ атомной плоскости (рис. 8), равна

$$
\Delta R=\frac{x^{2} \sin ^{2} \vartheta+y^{2}}{2 R_{1}}+\frac{x^{2} \sin ^{2} \vartheta+y^{2}}{2 R_{2}},
$$

где $R_{1}$ - расстояние фокус рентгеновской трубкикристалл, $R_{2}$ - расстояние кристалл-точка наблюдения. Граница первой зоны Френеля определяется условием $\Delta R=\lambda / 2$. Тогда из приведенного выше выражения получим уравнение для первой зоны Френеля

$$
\lambda=x^{2} \sin ^{2} \vartheta\left(\frac{1}{R_{1}}+\frac{1}{R_{2}}\right)+y^{2}\left(\frac{1}{R_{1}}+\frac{1}{R_{2}}\right) .
$$

Это уравнение показывает, что первая зона Френеля представляет собой эллипс, вытянутый вдоль оси $x$. Величины полуосей $a$ и $b$ равны соответственно

$$
a=\frac{1}{\sin \vartheta} \sqrt{\frac{R_{1} R_{2}}{R_{1}+R_{2}} \lambda}, \quad b=\sqrt{\frac{R_{1} R_{2}}{R_{1}+R_{2}} \lambda} .
$$

Учитывая, что в традиционно используемой схеме съемки Брэгга-Брентано расстояние $R_{1}$ равно $R_{2}$ и в большинстве рентгеновских дифрактометров составляет 18-20 cm, мы получаем диаметр первой зоны Френеля, равный $\sim 1-3 \mu \mathrm{m}$. При переходе во вторую зону Френеля рассеяние рентгеновской волны происходит в противофазе рассеяния на первой зоне Френеля и, следовательно, суммарная интенсивность должна уменьшаться. Изменение амплитуды рассеяния $O A$ результирующей волны в зависимости от того, сколько зон Френеля перекрыто, описывается спиралью Корню [26] (рис. 9). В соответствии с оценкой, полученной по формуле (3), для частиц, меньших диаметра первой зоны Френеля, интенсивность рассеяния, согласно данным рис. 9, будет расти с увеличением размера, а при превышении этого размера интенсивность отражений начнет уменьшаться. Именно такой интервал размеров отображен на рис. 6 , и именно изменение размера в этом интервале при последовательных отжигах порошка $\mathrm{YBO}_{3}$ привело к зависимостям пиковой и интегральной интенсивностей рефлексов, показанным на рис. 5 .

\section{4. Заключение}

Приведенные результаты позволяют сделать два основных вывода. 1. На относительное распределение интенсивностей рентгеновских рефлексов в промежуточной области между нано- и микроразмерами значительное влияние оказывают размер и форма кристаллитов.

2. С помощью прямых рентгеновских исследований показано, что в ортоборате иттрия действительно образуется моноклинная модификация, но она реализуется при температурах выше $1000^{\circ} \mathrm{C}$.

Кроме того, исходя из формы спирали Корню можно заключить, что для размера кристаллитов, перекрывающих $\sim 10-12$ зон Френеля, интенсивность рефлексов выйдет на асимптотику и будет соответствовать обычной кинематической теории рассеяния рентгеновских лучей. Для используемой нами геометрии Брэгга-Брентано этот размер оценивается в 15-20 $\mu \mathrm{m}$.

Авторы выражают искреннюю благодарность Г.К. Струковой за помощь в получении аморфных прекурсоров $\mathrm{YBO}_{3}$ и Е.Ю. Постновой за проведение SEM-анализа. 


\section{Список литературы}

[1] I.M. Shmytko, G.K. Strukova, E.A. Kudrenko. Cryst. Rep. 51, Suppl. 1, S163 (2006).

[2] И.М. Шмытько, Е.А. Курденко, Г.К. Струкова. Письма в ЖЭТФ 86, 544 (2007).

[3] Е.А. Кудренко, И.М. Шмытько, Г.К. Струкова. ФТТ 50, 924 (2008).

[4] И.М. Шмытько, Е.А. Кудренко, Г.К. Струкова, Н.В. Классен. ФТТ 50, 1108 (2008).

[5] И.М. Шмытько, Г.К. Струкова. ФТТ 51, 1796 (2009).

[6] И.М. Шмытько, Е.А. Кудренко, Г.К. Струкова. ФТТ 51, 1834 (2009).

[7] I. Shmytko, G. Strukova, E. Kudrenko. Acta Cryst. A 66, 230 (2010).

[8] И.М. Шмытько, И.Н. Кирякин, Г.К. Струкова. ФТТ 53, 353 (2011).

[9] I. Shmytko. Acta Cryst. A 67, 533 (2011).

[10] V.V. Kedrov, I.M. Shmyt'ko, S.Z. Shmurak, E.A. Kudrenko, N.V. Klassen. J. Mater. Res. 27, 2117 (2012).

[11] И.М. Шмытько, И.Н. Кирякин, Г.К. Струкова. ФТТ 55, 1369 (2013).

[12] E.A. Kudenko, I.M. Shmytko, G.K. Strukova. Acta Cryst. A 64, 427 (2008).

[13] I.M. Shmytko, E.A. Kudrenro, G.K. Strukova, V.V. Kedrov, N.V. Klassen. Z. Krist. (Suppl.) 27, 211 (2008).

[14] E.A. Kudrenro, I.M. Shmytko, V.V. Synytzin, E.G. Ponyatovsky, B.S. Red'kin. Z. Krist. (Suppl.) 27, 205 (2008).

[15] E.M. Levin, C.R. Robbins. J. Waring. J. Am. Ceram. Soc. 44, 87 (1961).

[16] E.M. Levin, R.S. Roth, J.B. Martin. J. Am. Miner. 46, 1030 (1961).

[17] E.M. Levin. J. Am. Ceramic Soc. 50, 53 (1967).

[18] E.M. Levin. In: Phase diagrams / Ed. A.M. Alper. Academic, N.Y. (1970). V. 3, P. 180.

[19] Y.H. Wang, J.C. Zhang, L. He, X. Guo, L.L. Wang, C.F. Wu. Mater. Res. Soc. Symp. Proc. 878E, 4.7.1 (2005).

[20] Z.-G. Wei, L.-D. Sun, C.-S. Liao, X.-C. Jiang, C.-H. Yan. J. Mater. Chem. 12, 3665 (2002).

[21] J. Plewa, T. Jüstel. J. Thermal Analys. Calorim. 88, 531 (2007).

[22] S. Hosokawa, Y. Tanaka, S. Iwamoto, M. Inoue. J. Mater. Sci. 43, 2276 (2008).

[23] H. Zhu, L. Zhang, T. Zuo, X. Gu, Z. Wanga, L. Zhu, K. Yao. Appl. Surf. Sci. 254, 6362 (2008).

[24] L. Lia, S. Zhoua, S. Zhanga. Solid State Sci. 10, 1173 (2008).

[25] J.H. Lin, D. Sheptyakov, Y.X. Wang, P. Allenspach. Chem. Mater. 16, 2418 (2004).

[26] В.И. Иверонова, Г.П. Ревкевич. Теория рассеяния рентгеновских лучей. Изд-во МГУ, М. (1972). 246 с. 